\title{
Thrombotic Thrombocytopaenic Purpura after Aortic Valve Replacement with Trifecta Bio-Prosthesis: A Case Report
}

\section{F Falconieri* and G Krasopoulos}

\author{
Cardiothoracic Registrar, John Radcliffe Hospital, Oxford University Hospital NHS Foundation Trust, Oxford, UK
}

\begin{abstract}
Thrombocytopaenia is a recognized complication after aortic valve replacement (AVR), sometimes it has been reported as heparin-induced, and it can be associated with high mortality and morbidity. This case report is describing the first case of thrombotic thrombocytopaenic purpura (TTP) after AVR using the Trifecta bioprosthesis. We recommend that patients with severe and progressive thrombocytopenia after biological AVR should be screened early for both TTP and Heparin Induced Thrombocytopenia (HIT), in an attempt to shorter the decision-making process, provide appropriate therapy to the patients and reduce any associated mortality and morbidity.
\end{abstract}

\section{Introduction}

Thrombocytopenia after cardiac surgery is associated with the type of surgery performed, the length of time that the patient stays on extracorporeal circulation [1] and pharmacologic treatment during the first postoperative hours [2]. Thrombocytopaenia after aortic valve replacement (AVR) has been reported by several authors [3-6] sometimes reported as heparin-induced [7] and it is associated with high mortality and morbidity. This case report describes the first case of thrombotic thrombocytopaenic purpura (TTP) after AVR with the use of Trifecta (St Jude Medical, Little Canada, Minnesota) bioprosthetic valve.

\section{Case Report}

A 66-year-old female was referred to us for aortic valve surgery after she has been suffering from shortness of breath for few months. A transthoracic echocardiogram confirmed the presence of severe aortic valve stenosis on background of mildly impaired left ventricular function and her past medical history included one episode of transient ischemic attack and new onset of atrial fibrillation; therefore, she was started on LMWH (low molecular weight heparin) for a period of 2 weeks before her operation date. Her preoperative hemoglobin (Hb) was $136 \mathrm{mg} / \mathrm{dL}$ and platelets (PLTs) count was 253 $\times 10^{9} / \mathrm{L}$.

She underwent biological AVR with a $23 \mathrm{~mm}$ St. Jude Medical Trifecta bioprosthesis. The operation was uneventful, and the postoperative echocardiogram confirmed a well seated, well-functioning AVR prosthesis with no evidence of peri-prosthetic leak.

On the second postoperative day (POD 2) the PLTs count started dropping $\left(125 \times 10^{9} / \mathrm{L}\right)$. By POD 4 her platelet count has dropped further to $52 \times 10^{9} / \mathrm{L}$, despite the discontinuation of LWMH. Hb remained stable at $83 \mathrm{mg} / \mathrm{dl}$. On POD 5 there was a further drop of the platelet count to 33 and following haematologist advice, the patient was started on Fondaparinux. HIT (Heparin-induced Thrombocytopenia) screening (ELISA test) was performed and after it was confirmed negative, Fondaparinux was stopped and one pool of PLTs was then transfused. As the PLTs count had further dropped to $22 \times 10^{9} / \mathrm{L}$ the patient was screened for TTP which included Von Willebrand protease (ADAMTS13), Von Willebrand inhibitor levels, Coomb test, Blood smear and LDH. While the test results were awaited the pa-

${ }^{*}$ Corresponding author: F Falconieri, Cardiothoracic Registrar, John Radcliffe Hospital, Oxford University Hospital NHS Foundation Trust, Headley Way, Headington, Oxford, UK, Tel: +44-(0)-7729924285, E-mail: ffalconieri@hotmail. com

Received: May 24, 2017; Accepted: June 18, 2018; Published online: June 20, 2018

Citation: Falconieri F, Krasopoulos G (2018) Thrombotic Thrombocytopaenic Purpura after Aortic Valve Replacement with Trifecta Bio-Prosthesis: A Case Report. J Cardiothorac Surg Ther 2(1):17-19

Copyright: (C) 2018 Falconieri F, et al. This is an open-access article distributed under the terms of the Creative Commons Attribution License, which permits unrestricted use, distribution, and reproduction in any medium, provided the original author and source are credited. 
Citation: Falconieri F, Krasopoulos G (2018) Thrombotic Thrombocytopaenic Purpura after Aortic Valve Replacement with Trifecta Bio-Prosthesis: A Case Report. J Cardiothorac Surg Ther 2(1):17-19

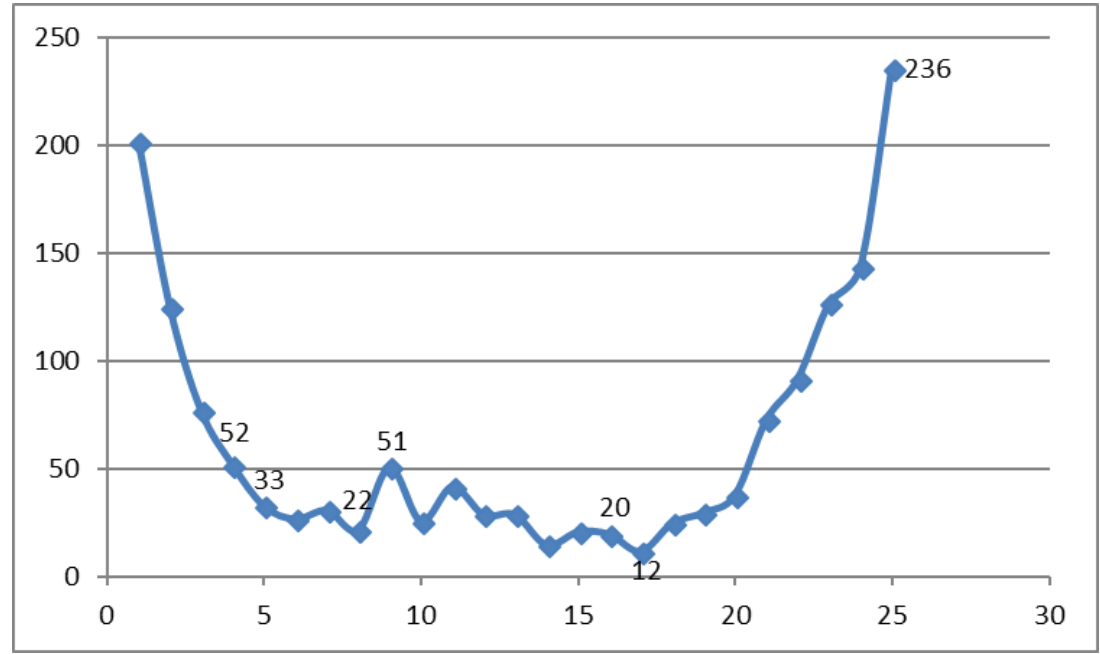

Figure 1: The graph shows the postoperative platelet count fluctuation in relation to postoperative days (POD) and therapeutic interventions; 52: POD4 PLTs count 52 (LMWH stopped); 33: POD5 PLTs count 33 (HIT screening sent); 22 : POD8 PLTs count 22 (ELISA results false negative, Fondaparinux stopped,1 pool PLTs transfused); 51: POD9 vWs protease (ADAMTS13), vWs inhibitor, Coombs, LDH sent, POD16 TTP diagnosis confirmed; 20: POD17 (Plasma Exchange × 6); 236: POD25 (PLTs count normalised).

tients PLTs count dropped to $12 \times 10^{9} / \mathrm{L}$, despite further transfusion of two more pools of PLTs. Two weeks after surgery the diagnosis of TTP was confirmed, the patient underwent plasma exchange (PEX). In plasma exchange, the removed plasma was discarded, and the patient received replacement donor plasma, with quick recovery of her PLTs count to the baseline level. The patient was discharged home with PLTs counting at $230 \times 10^{9} / \mathrm{L}$. The patient was followed up in our outpatient department and she was found to be clinically well with normal count of PLTs (Figure 1).

\section{Discussion}

After cardiac surgery platelets count can drop significantly. As described in literature, this is mainly caused by the type of the operation and its complexity, the length of time on cardiopulmonary bypass and anti-coagulation therapy during the first postoperative hours $[1,2]$. Cases of thrombocytopaenia after aortic valve replacement (AVR) has been reported by several authors and sometimes reported as heparin-induced, with associated mortality and morbidity $[6,7]$.

In 2009 Thielmann, et al. [7] published a study where postoperative cardiac surgical patients were screened for HIT antibodies, when PLTs dropped to less than $50 \%$ of the baseline level or less than $50 \times 10^{9} / \mathrm{L}$ and compared the outcomes in HIT-antibody-positive and HIT-antibody-negative patients. As result they found that patients in whom a low platelet count prompted testing for HIT antibodies had a high mortality, independent of whether heparin-dependent antibodies were present, indicating that a persistently lowered platelet count is a bad prognostic sign after cardiac surgery.
Hilker, et al. [3] described thrombocytopenia following AVRs with Carpentier Edwards Perimount and Sorin Biomedica Freedom SOLO bioprosthesis. A slower recovery of PLTs counts in the SOLO-group was seen for all used valve sizes and it was not related to the duration of bypass time nor with patient's age. The authors speculated that the observed thrombocytopenia was due to transient, unspecific activation of platelets which led to a diffuse consumption and lower platelet levels, but they could not comment on real cause or the clinical implications of this phenomenon.

Van Straten, et al. [5] have looked into thrombocytopaenia following AVR with biological and mechanical prosthesis. They found that patient after AVR with Carpentier-Edwards Perimount and Medtronic Freestyle stentless bioprosthesis had significantly lower postoperative platelet count in comparison to patients receiving ATS and St. Jude Medical mechanical prostheses; while in their analysis there was no difference identified between the Sorin Freedom Solo and all other valve prostheses.

Ravenni, et al. [6] have observed postoperative thrombocytopaenia after AVR with Sorin Freedom Solo, Medtronic Mosaic and the Sorin Mitroflow bioprosthesis, which was more pronounced for the Sorin Freedom Solo valve. Their observed thrombocytopenia after AVR with biological prostheses was not associated with any major postoperative complication.

At the best of our knowledge, low PLTs count has never been described as secondary to thrombotic thrombocytopenic purpura (TTP) after AVR with SJM Trifecta bioprosthesis. In this case report and following the diag- 
Citation: Falconieri F, Krasopoulos G (2018) Thrombotic Thrombocytopaenic Purpura after Aortic Valve Replacement with Trifecta Bio-Prosthesis: A Case Report. J Cardiothorac Surg Ther 2(1):17-19

nosis of thrombocytopaenia, the patient underwent first a HIT and TTP screening was in sequence leading to a considerable delay of the diagnosis of TTP, a rear and potentially dangerous complication that can be easily treated if diagnosed at an early stage.

With this case report we would like to not only report the first case of TTP after AVR using the Trifecta bioprosthesis but also to recommend that patients with severe and progressive thrombocytopenia after biological AVR (and maybe any cardiac surgical procedure) are screened early for TTP along with HIT, in an attempt not only to shorter the decision-making process but also to provide appropriate therapy to the patient.

\section{Conflict of Interest}

None.

\section{References}

1. Weerasinghe A, Taylor KM (1998) The platelets in cardiopulmonary bypass. Ann Thorac Surg 66: 2145-2152.
2. Le Guyader A, Watanabe R, Berbe J, et al. (2006) Platelet activation after aortic prosthetic valve surgery. Interact Cardiovasc Thorac Surg 5: 60-64.

3. Hilker L, Wodny M, Ginesta M, et al. (2009) Differences in the recovery of platelet counts after biological aortic valve replacement. Interact Cardiovasc Thorac Surg 8: 70-73.

4. Piccardo A, Rusinaru D, Petitprez B, et al. (2010) Thrombocytopenia after aortic valve replacement with freedom solo bio-prosthesis: A propensity study. Ann Thorac Surg 89: 1425-1430.

5. Van Straten AH, Hamad MA, Berreklouw E, et al. (2010) Thrombocytopenia after aortic valve replacement: Comparison between mechanical and biological valves. J Heart Valve Dis 19: 394-399.

6. Ravenni G, Celiento M, Ferrari G, et al. (2012) Reduction in platelet count after aortic valve replacement: Comparison of three bioprostheses. J Heart Valve Dis 21: 655-661.

7. Thielmann M, Bunschkowski M, Tossios P, et al. (2010) Perioperative thrombocytopenia in cardiac surgical patients-incidence of heparin-induced thrombocytopenia, morbidities and mortality. Eur J Cardiothorac Surg 37: 1391-1395 